\title{
MedienPädagogik
}

www. medienpaed.com

Zeitschrift für

Theorie und Praxis

der Medienbildung

ISSN 1424-3636

\section{Kreativität fördern durch körperbasiertes Lernen}

\section{Pragmatistische Perspektiven für den Unterricht in Schule und Universität}

Alexander Gröschner und Mike Sandbothe

The question of the integration of mind-body in action is the most practical of all questions we can ask of our civilization. (John Dewey, 1984, S. 29)

\begin{abstract}
Vor dem Hintergrund des aktuellen Stands der internationalen Kreativitätsforschung untersucht der Beitrag die Rolle des menschlichen Körpers als mehrdimensionales Medium innerhalb von Lehr- und Lernprozessen. Zu diesem Zweck wird aus pragmatistischer Perspektive ein integraler Ansatz körperbasierten Lernens skizziert. Darüber hinaus zeigen die Autoren am Beispiel von universitären Seminarveranstaltungen, die sie in Deutschland und Dänemark durchgeführt haben, wie body-based learning in die Lehr- und Lernkultur an Schulen und Universitäten eingebettet werden kann.
\end{abstract}

\section{Einleitung}

In einer Zeit, in der die Internet-Suchmaschine Google weltweit zu den am häufigsten gewählten Informationsquellen gehört und zwischenmenschliche personale Interaktion zunehmend auch via SMS, E-Mail, Twitter oder social networking sites wie Facebook und MySpace stattfindet, tritt im Kontext von Lehr- und Lernprozessen zugleich ein lange verdrängtes altes Medium in den Fokus der Betrachtungen: der menschliche Körper.

Seine pädagogische Revalidierung macht Sinn. Denn das neue Leitmedium Internet setzt als Bildschirmtechnologie die Vernachlässigung körperlicher Bewegung in unseren Bildungsinstitutionen fort. Das verbindet die Nutzung von Computer und Internet im Unterricht mit der didaktischen Integration der audiovisuellen Massenmedien (Radio, Kino, Video, Fernsehen), die in der zweiten Hälfte des zwanzigsten Jahrhunderts in unseren Schulen und Universitäten stattgefunden hat. Beide Prozesse verstärken die Tendenz zur pädagogischen Sitzkultur, die sich seit dem Zeitalter des Buchdrucks im modernen Bildungssystem etabliert hat. Die Wiederentdeckung des Körpers in der zeitgenössischen Bildungsarbeit (Glynn, 
2001; Bresler, 2004; Hannaford, 2005) erscheint vor diesem Hintergrund als eine ausbalancierende Gegenbewegung, die längst überfällig war (Sandbothe, 2010a). Im Folgenden möchten wir zeigen, dass der Körper als «our most primordial tool of tools» (Shusterman, 2008, S. 4) im Unterricht als Werkzeug zur Initiierung und Intensivierung kreativer Prozesse genutzt werden kann. Als körperbasiert bezeichnen wir in diesem Zusammenhang einen Ansatz, der nicht nur das Zusammenspiel von Skelett, Muskulatur und Organen mit unserem Gehirn und Nervensystem, sondern auch unser Denken und unsere Phantasie, unsere Gefühle und unsere Handlungen als integrale Dimensionen energetischer Körperprozesse im weitesten Sinne begreift (Alexander, 2001; Feldenkrais, 1992, 2005; Shusterman, 2008).

Körperbasiertes Lehren und Lernen in diesem integralen Verständnis hilft Studierenden, Schülerinnen und Schülern dabei, ihre ganzheitliche Selbst- und Fremdwahrnehmung zu schulen. Auf diesem Weg kann es zu verbesserten Selbstregulationsprozessen kommen, die ihrerseits weit reichende Potenziale für die Freisetzung von neuen Dimensionen kreativen Empfindens, Denkens und Handelns eröffnen.

Bei unseren Ausführungen gehen wir in drei Schritten vor:

- Zunächst werden wir auf die Entwicklung und den aktuellen Stand der internationalen Kreativitätsforschung eingehen und exemplarisch vor Augen führen, inwiefern diese sich bei der Bestimmung des Kreativitätsbegriffs bisher vor allem an geistigen Fähigkeiten und intellektuellen Tugenden ausgerichtet hat.

- Im zweiten Teil kennzeichnen wir den menschlichen Körper als mehrdimensionales Medium der Wahrnehmung, der Kommunikation und des Handelns. Vor diesem Hintergrund bringen wir bildungsphilosophische Ansätze in den Blick, die im Kontext des amerikanischen Pragmatismus von William James (18421910) und John Dewey (1859-1952) entwickelt worden sind. Diese Ansätze werden von uns genutzt, um den Körper im Lehr- und Lernprozess zu verorten und den Kreativitätsbegriff körperphilosophisch zu erweitern.

- Drittens präsentieren wir den Ansatz des körperbasierten Lehrens und Lernens in Aktion. Das geschieht anhand von zwei exemplarischen Seminarkonzeptionen, die wir in Deutschland und Dänemark an verschiedenen Universitäten erprobt haben.

\section{Begriffliche Zugänge zu Kreativität und ihre Bedeutung für Lehr- und Lernprozesse}

Der Begriff Kreativität geht zurück auf das lateinische creator (Schöpfer, Erzeuger, Gründer) und das Verbum creare (erschaffen, zeugen, ins Leben rufen, auswählen), das seinerseits mit dem Verb crescere (wachsen, zunehmen, entstehen) eng verwandt ist. Im heutigen Begriffsverständnis wurde das Wort aus dem Englischen creativity in die deutsche Sprache übernommen (Serve, 1995, S. 20). 
Der Durchbruch in der interdisziplinären Erforschung des Phänomens der Kreativität gelang 1950 mit einem Vortrag von Joy Paul Guilford auf einem Kongress der American Psychological Association. Guilford trat damals für eine systematische und experimentelle Forschung auf dem Feld der Kreativität ein. Er ging davon aus, dass die Fähigkeit zum kreativen Handeln kein genetisch vorgegebenes Geschenk an spezifische Personengruppen (z. B. Familien mit künstlerischen Berufen), sondern vielmehr jedem Individuum auf seine Weise zugänglich ist: «Creative acts can therefore be expected, no matter how feeble or how infrequent, of almost all individuals» (Guilford, 1950, S. 450; vgl. Guilford, 1970).

Entgegen der Ansicht, dass Kreativität als Forschungsthema eine eher kurzlebige Modeerscheinung der letzten 50 Jahre sei, ist festzustellen, dass sich Überlegungen zur menschlichen Kreativität in der Geschichte des abendländischen Denkens von Platon über F.W. J. Schelling bis in die Gegenwart finden. Während Platon die schöpferischen Kräfte von Philosophen und Dichtern in ihrer Fähigkeit zum Enthusiasmus vermutete, stellte Schelling heraus, dass der Mensch erst durch schöpferisches Handeln zu dem wird, was er ist (vgl. Serve, 1995, S. 22f.). In der Pädagogik waren es vor allem Pestalozzi und Fröbel im 19. Jahrhundert sowie die Vertreter der reformpädagogischen Bewegung Ende des 19., Anfang des 20. Jahrhunderts wie u. a. Dewey, Freinet und Reichwein, die das schöpferische Zusammenspiel von Körper, Seele und Geist bei der Erziehung und bei Lernvorgängen des kindlichen Individuums betont haben (vgl. Gröschner, 2005).

Wie Sternberg und Lubart (1999) in dem von Sternberg herausgegebenen Handbook of Creativity einführend festhalten, hat sich das Forschungsfeld in praktischer, theoretischer und methodologischer Hinsicht seit Guilford deutlich ausgeweitet, so dass eine übergreifende Charakterisierung der Kreativitätsforschung nicht leicht zu erstellen ist (vgl. hierzu auch Feldman \& Benjamin, 2006). Es fällt jedoch auf, dass die körperbasierte Perspektive - wie wir sie im zweiten Teil unserer Überlegungen skizzieren - in der Kreativitätsforschung bislang nicht ausreichend entwickelt worden ist. Ausserhalb der reformpädagogisch inspirierten Forschungsparadigmen überwiegen Ansätze, die auf die Förderung der geistigen Fähigkeiten und intellektuellen Tugenden der Lernenden abzielen, ohne dabei deren körperliche Fundierung angemessen zu berücksichtigen (vgl. hierzu auch kritisch Hentig, 1998).

So identifizieren etwa Sternberg und Lubart die folgenden drei geistig-intellektuellen Kompetenzen im Zusammenhang mit Kreativität: «(i) [The] analytic ability to recognize which of one's ideas are worth pursuing and which are not; (ii) the synthetic ability to see problems in new ways and escape the bounds of conventional thinking: and (iii) the practical-contextual ability to realize new ideas and persuade others of their value» (Sternberg \& Lubart, 1999, S. 11). Die analytische Fähigkeit zu erkennen, welche der eigenen Ideen es wert sind, weiter verfolgt zu werden, ist eine Leistung der bestimmenden Urteilskraft. Die synthetische Fähig- 
keit, Probleme auf neue Weise zu sehen und die Grenzen des konventionellen Denkens zu überschreiten, setzt reflektierende Urteilskraft bzw. Phantasie voraus. Und die Fähigkeit, neue Ideen in konkreten Kontexten umzusetzen und andere Menschen von ihrer Bedeutung zu überzeugen, erfordert Einfühlungsvermögen, Überzeugungskraft, Erfahrung und lebenspraktische Klugheit, d. h. phronesis im aristotelischen Sinn.

Alle drei geistig-intellektuellen Fähigkeiten bzw. Tugenden beinhalten grundsätzlich einen selbstreflexiven Aspekt der individuellen Wahrnehmung, wie er insbesondere aus einem Verständnis von Kreativität als Teil der Persönlichkeit erwächst. Im Kontext von Lehr- und Lernprozessen ist diese Perspektive als wichtige Teilkomponente aufzufassen. Sie betont, dass individuelle Fähigkeiten und Einstellungen das kreative Potenzial einer Person beeinflussen. Dabei spielen sowohl Wissen als Voraussetzung kreativen Handelns als auch motivationale Aspekte (vor allem intrinsische Motivation) eine entscheidende Rolle für den Unterricht in Schule und Universität (Cropley, 2001; Csikszentmihalyi, 1997). Auch die pädagogische Innovationsforschung betont diese individuellen Fähigkeiten, motivationalen Orientierungen und Einstellungen (z. B. Offenheit für neue Erfahrungen) als Ausgangspunkte für nachhaltige Veränderungen (Gröschner, im Druck).

Für den Unterricht sind darüber hinaus zwei weitere Aspekte von Kreativität besonders bedeutsam: der kreative Prozess und das kreative Produkt (Preiser \& Buchholz, 2004; Goleman, Kaufman \& Ray, 1997).

Der kreative Prozess ist vergleichbar mit dem Problemlösungsprozess. Um einen als unbefriedigend empfundenen Zustand zu verändern bzw. einen verbesserten Zielzustand zu erreichen, ist es notwendig, das Problem zunächst zu erkennen und anschliessend nach geeigneten Lösungen zu suchen. Fudickar betont im Anschluss an Guilford als Voraussetzung für einen kreativen Lösungsprozess die Fähigkeit zum divergierenden Denken:

Die wichtigste Voraussetzung für kreatives Verhalten ist das divergierende Denken während des kreativen Prozesses; divergierendes Denken sucht jeweils nach mehreren Lösungen für ein Problem, während das konvergierende Denken ein Problem unter Anwendung herkömmlicher Denkvorstellungen und Regeln zu lösen vermag. (Fudickar, 1985, S. 36)

Für die Erhöhung der Intensität divergierender Denkstrategien erachtet u. a. Egger im Kontext des Unterrichts den sozialen Austausch im Klassenraum für bedeutsam. Durch gezielte kommunikative Prozesse unter den Schülerinnen und Schülern, die eine klare Strukturiertheit des Arbeitsauftrages voraussetzen, können in kurzer Zeit im Unterricht wünschenswerte Ergebnisse - d. h. Lösungswege - erreicht werden (Egger, 1996, S. 28ff.). Auch Cropley hebt aus pädagogisch-psychologischer Sicht kommunikative Aspekte im Klassenkontext hervor. Er betont insbesondere die Rol- 
le der Lehrperson, die im sozialen Kontext kreativitätshemmende Bedingungen abbauen kann (Cropley, 2001, S. 269).

Betrachtet man Möglichkeiten der Förderung von Kreativität im schulischen und universitären Handlungsfeld, so wird deutlich, dass sowohl die individuelle Persönlichkeit als auch die Rahmenbedingungen für den kreativen Prozess - die Lernumgebung - massgebliche Einflussfaktoren für kreatives Denken und Handeln darstellen. Aus diesem Grund wird im Kontext der Kreativitätsforschung gefordert, die schulischen und universitären Lernumgebungen so zu gestalten, dass kommunikative und kooperative Prozesse zwischen den Lernenden durch sie unterstützt werden (Loi \& Dillon, 2006; Neff, 1975). Dies setzt mitunter weniger frontale als vielmehr interaktive Raumstrukturen und Sozialformen in Unterricht und Lehre voraus, die zudem die intrinsische Motivation und das Interesse der Lernenden am Gegenstand berücksichtigen und ggf. sogar erhöhen (Weiss, 2008; Seidel et al., 2009).

Das kreative Produkt wird unterschiedlich definiert und in der Kreativitätsforschung kontrovers diskutiert. Zum einen kann das Ergebnis eines kreativen Denkprozesses - ein neuer Gedanke - darunter verstanden werden. Häufiger wird mit dem Wort Kreativität aber auch eine materielle Innovation verbunden, die vor allem im Bildungsbereich erschwerten Bedingungen der Umsetzung unterworfen ist (Reinmann, 2005; Cropley, 2001; Gröschner, im Druck).

Anknüpfend an die bisherigen Ausführungen können vier Dimensionen für die Förderung von Kreativität im Unterricht gekennzeichnet werden, die auf den drei dargestellten Aspekten (Persönlichkeit, Prozess, Produkt) aufbauen (vgl. Jeffrey, 2006):

- Relevanz: Lernen ist bedeutsam für die konkreten Bedürfnisse der Schülerinnen und Schüler sowie für die Gesamtinteressenlage der Gruppe als Lerngemeinschaft.

- Bewusstheit von Wissen: Die Schülerinnen und Schüler lernen für sich selbst - nicht für Lehrer, Leistungskontrollen oder bestimmte gesellschaftliche Anforderungen. Kreatives Lernen ist internalisiert; es standardisiert nicht, sondern unterscheidet die individuellen Persönlichkeiten voneinander.

- Selbstkontrolle: Schülerinnen und Schüler lernen selbstmotiviert. Die Kontrolle von Lernprozessen erfolgt nicht in erster Linie durch extrinsische Faktoren oder aufgabenorientierte Übungen.

- Innovation: Dem Entstehen von Neuem Raum zu geben, ist ein zentrales Lernziel kreativer Unterrichtsformen. So können z. B. eine Fähigkeit verbessert, neue Einsichten oder neues Wissen gewonnen oder ein neues Produkt hergestellt werden. Die Orientierung am Prozess der Veränderung schafft ein stärker graduelles und kumulatives Lernverständnis. 
Die für den schulischen Unterricht ausdifferenzierten Dimensionen sind auch für die universitäre Lehre bedeutsam. Zugleich korrespondieren diese Schlüsseleigenschaften mit einem konstruktivistischen, kognitiv aktivierenden und reflektierten Lehr- und Lernverständnis, wie es in der neueren Schul- und Unterrichtsforschung vertreten wird (Reinmann \& Mandl, 2006; Renkl, 2008; Scheerens \& Bosker, 1997; Shuell, 1996).

Serve definiert Kreativität darüber hinaus als eine «aus mehreren Komponenten bestehende, nicht nur geistig, sondern auch körperlich und gefühlsmässig bedingte Begabung, Tendenz und Lebenskraft des Menschen» (Serve, 1995, S. 43). Das bedeutet, dass Kreativität nicht nur mit kognitiven Fähigkeiten und intellektuellen Tugenden zu tun hat, sondern das Zusammenspiel von körperlich-sensuellen, seelisch-affektiven und geistig-kognitiven Dimensionen im Prozess kreativen Lehrens und Lernens eine wichtige Rolle spielt (Weiss, 2008, S. 131).

Zur Förderung kreativer Lernprozesse plädieren wir in den weiteren Ausführungen für einen pragmatistischen Ansatz körperbasierten Lernens als Instrument zur Verbesserung der eigenen Körperwahrnehmung und zur Stärkung von Selbstregulationsprozessen. Bevor wir dazu zwei von uns erprobte Seminarkonzepte vorstellen, bringen wir den menschlichen Körper als mehrdimensionales Medium in den Blick und führen seine grundlegende Bedeutung für kreative Lehr- und Lernprozesse im Rekurs auf William James und John Dewey vor Augen.

\section{Der menschliche Körper als mehrdimensionales Medium des Lehrens und Lernens}

Den menschlichen Körper als Medium zu betrachten, setzt voraus, dass man den Medienbegriff nicht - wie in der Medien- und Kommunikationswissenschaft lange Zeit üblich - auf die technische Dimension massenmedialer Kommunikation beschränkt. Orientiert man sich stattdessen an einem weiten Verständnis des Wortes, lassen sich Medien als Steuerungsinstrumente auffassen, mit deren Hilfe sich eine bewusste oder unbewusste Veränderung von Wirklichkeit intra- und/oder intersubjektiv koordinieren lässt.

Mike Sandbothe und Ludwig Nagl haben in der von ihnen herausgegebenen Systematischen Medienphilosophie (2005) den Vorschlag gemacht, die unterschiedlichen Verwendungsweisen des Wortes Medium wie folgt zu differenzieren: a) Sinnliche Wahrnehmungsmedien wie z. B. Raum und Zeit, b) Semiotische Kommunikationsmedien wie z. B. Bild, Sprache, Schrift und Musik sowie c) Technische Verbreitungsmedien wie z. B. Buchdruck, Radio, Film, Fernsehen, Computer und Internet. Bereits in einer früheren Publikation hat Sandbothe mit Blick auf diesen Systematisierungsvorschlag hervorgehoben: 
Bei den genannten Beispielen handelt es sich jeweils um offene Reihen ohne Anspruch auf Vollständigkeit. So kommen neben den Anschauungsformen von Raum und Zeit im Bereich der sinnlichen Wahrnehmungsmedien insbesondere die Sinnesorgane mit ins Spiel. Entsprechend sind zu den technischen Verbreitungsmedien die Artikulationsorgane, aber auch der Körper (...) zu rechnen. Und die semiotischen Kommunikationsmedien umfassen neben den exemplarisch genannten auch die Zeichensysteme der Gerüche, der Geschmäcke, der Berührung sowie Gestik, Mimik oder Tanz. (Sandbothe, 2003, S. 189)

Betrachtet man diesen "gebrauchstheoretischen» (Sandbothe, 2005, S. XV) Ansatz genauer, dann wird deutlich, dass unterschiedliche Nutzungsformen und Betrachtungsweisen des menschlichen Körpers in allen drei Bereichen auftauchen: Sinnesorgane, Gestik und Mimik sowie der anatomisch oder biologisch verstandene Organismus insgesamt lassen sich im weiten Medienverständnis in unterschiedlichen Kontexten verorten. Hilfreich erscheint dieser Ansatz vor allem in der Hinsicht, dass er den menschlichen Körper als Medium nicht starr zuordnet, sondern eine funktionale Differenzierung zulässt, welche die sinnlichen, semiotischen und technischen Dimensionen unseres medialen Körpergebrauchs zueinander ins Verhältnis setzt.

Vor diesem Hintergrund erscheint der menschliche Körper als ein zentrales Koordinationsmedium, das im Hinblick auf Bildungs- und Erziehungsprozesse eine wichtige Rolle spielen kann (Hoffmann, 2003). Der Körper ist in einer durch Faceto-face-Kommunikation geprägten Lehr- und Lernkultur das multidimensionale Instrument der Vermittlung und Integration von erziehungsrelevanten Werten und Wissensbeständen. Aus diesem Grund plädiert Gröschner an anderer Stelle für eine Revalidierung des Körpers im Kontext der Unterrichtskommunikation; eine Revalidierung, die neben der Bedeutung der Etablierung einer sprachlich orientierten Rückmeldekultur für die Berücksichtigung nonverbaler Aspekte (z.B. als Beitrag zur Klassenführung) eintritt (Gröschner, 2007).

Die medienpädagogische Bedeutsamkeit des Körpers wird im Kontext von Bildung und Erziehung oftmals nicht ausreichend wahrgenommen (vgl. hierzu auch Giesecke, 2007). Bereits Dewey hat das westliche Erziehungssystem in dieser Hinsicht kritisiert. Aus seiner Sicht legt es den Fokus zu stark auf kognitive Prozesse und Leistungen und zu wenig darauf, Kinder, Jugendliche und Erwachsene darin zu unterrichten, ihren Körper bewusst wahrzunehmen und intelligent als Medium in Kommunikation und Handlung einzusetzen. In der Einführung zu Frederick M. Alexanders Buch Der Gebrauch des Selbst (engl. zuerst 1932) schreibt Dewey: 
Erziehung ist die einzig sichere Methode, mit der die Menschheit ihren eigenen Kurs bestimmen kann. Doch wir sind in einem Teufelskreis gefangen, denn ohne zu wissen, was ein wahrhaft normales, gesundes psycho-physisches Leben ist, kommt das, was wir für Erziehung halten, eher einer Fehlerziehung gleich. (Dewey, 2001, S. XVIII)

Im Anschluss an Dewey hat Richard Shusterman den Körper als mediales Werkzeug der Koordination von wirklichkeitsverändernden Handlungen beschrieben: «[The] body must be recognized as our most primordial tool of tools, our most basic medium for interacting with our various environments, a necessity for all our perception, action, and even thought» (Shusterman, 2008, S. 4). Tatsächlich gilt, dass der Körper in mehrfacher Hinsicht als mediales Werkzeug beschrieben werden kann. Zum einen vermittelt er die Interaktion mit den verschiedenen Umwelten, in denen sich der Mensch bewegt. In einem rudimentären Sinn ist Interaktion mit der Umwelt zunächst Bewegung des menschlichen Körpers und seiner Teile im Raum (Pikler, 2001). Diese wiederum ist die Voraussetzung für aktives Handeln, d. h. für Prozesse der Veränderung der äusseren Wirklichkeit durch kausale bzw. physische Intervention. Wahrnehmung und Denken fungieren als Elemente dieses grundlegenden Interaktionsprozesses, der für das kindliche Individuum lebensnotwendig ist.

Zum anderen erscheint der Körper als Medium der funktionalen Koordination von Wahrnehmen, Fühlen, Denken und Handeln. Das Zusammenspiel dieser unterschiedlichen Ebenen des menschlichen Selbst wird sowohl individual- als auch gattungsgeschichtlich im Kontext der Einübung körperlicher Bewegung im Raum erlernt und kann daher im bewussten Rückgang auf seinen evolutionären Entstehungskontext auch modifiziert, intensiviert und kreativ weiterentwickelt werden. Darin liegt die besondere pädagogische Paradigmenfunktion von integralen Körper/Seele/Geist-Lerntechniken wie der Feldenkrais-Methode oder der AlexanderTechnik (Mathews, 1984; Friedmann, 1989; Shelav-Silberbusch, 1999).

Die Betrachtung des Körpers als mediales Werkzeug und als methodischer Gegenstand eines ganzheitlich verstandenen Lehrens und Lernens hat in der modernen Bildungsphilosophie eine lange reformpädagogische Tradition. Unter anderem haben Vertreter des amerikanischen Pragmatismus wie William James und John Dewey den Körper als Ausgangspunkt des Lernens durch das aktive Tun in dieser Weise aufgefasst. Indem sie dies taten, haben sie zugleich damit begonnen, körperliche Lernprozesse als Ausgangspunkte eines kreativen Selbst- und Welterlebens zu betrachten.

Für James besteht die Aufgabe des Lehrers darin, den Schüler in seinen Handlungen zu unterstützen «as including every possible sort of fit reaction on the circumstances into which he may find himself brought into by the vicissitudes of life» (James, 1983, S. 26). Lehrerinnen und Lehrer ermöglichen ihren Schülerinnen 
und Schülern Neues zu lernen, indem sie gemeinsam mit diesen in Handlungskontexten Erfahrungen machen und bewusst reflektieren. Für James ist Lernen ein Prozess, der aktives Problemlösen erfordert. Hat man sich mit einem Gegenstand oder Sachverhalt intensiv und aktiv auseinandergesetzt und Neues mit Bekanntem verknüpft, entsteht Wissen. Daraus folgt wiederum, dass Wissen niemals beendet oder statisch ist. James schreibt: «Knowing is a process of inquiring, not a static state» (James, 1970, S. 12).

Insofern kann man sagen, dass für James inhaltliches Wissen nicht in erster Linie ein vorgegebener Lernstoff ist, sondern strukturell das Produkt eines kreativen Problemlösungsprozesses. Mehr noch: Aus seiner Sicht sind Denken und Wissen als solche kreative Produkte eines evolutionären Problemlösungsprozesses. Weil der Mensch sonst nicht überlebensfähig wäre, nutzt er die Plastizität seines Nervensystems, um Wahrnehmung und Denken als miteinander verflochtene Interpretationsprozesse zu organisieren, deren gemeinsames Koordinatensystem durch den Körper als grundlegendes Medium der Interaktion konstituiert wird: "Where the body is is 〈here); when the body acts is 〈now»; what the body touches is «this»» (James, 1976, S. 86).

An verschiedenen Stellen seines Werks geht James sogar noch einen Schritt weiter, wenn er die physiologischen Grundlagen unserer seelischen und geistigen Funktionen mit Blick auf den menschlichen Körper wie folgt beschreibt: «lts breathing is my (thinking), its sensorial adjustments are my (attention), its kinaesthetic alterations are my (efforts), its visceral perturbations are my <emotions)» (James, 1976, S. 76). Aus heutiger Sicht ist die identifizierende Gleichsetzung emotionaler, motivationaler und mentaler Prozesse mit bestimmten Körperfunktionen sicherlich kritisch zu betrachten. Das relative Recht von James' Überlegung aber besteht darin, dass die seelisch-geistigen Aspekte unseres Selbst tatsächlich eng mit körperlichen Prozessen verbunden sind, ohne jedoch darauf reduziert werden zu können.

Diesem Zusammenhang ist James in seiner introspektiven Analyse körperlicher Empfindungen nachgegangen. Dabei hat er zu Recht darauf hingewiesen, dass sich die menschliche Fähigkeit zur propriozeptiven Innenwahrnehmung von Knochen, Muskeln, Gelenken und Organen in der technisierten Moderne kulturell zurück entwickelt hat. Das spiegelt sich auch in der Ontogenese. Jede Bewegung, die wir machen, ist in unserer frühen Kindheit einmal erlernt worden und daher mit Gefühlen des Erfolgs und Misserfolgs, der Begeisterung und Frustration eng verbunden. Jede Bewegung, die wir erlernt haben, haben wir einmal als kreative Lösung eines konkreten Handlungsproblems individuell entwickelt, bevor sie sich dann als Bewegungsmuster automatisiert hat. Aus diesem Grund kann bewusste Körpererfahrung emotionale Räume eröffnen. Emotionen wiederum können Handlungen motivieren. Und Handlungen sind der Kontext, in dem materielle Produkte und mentale Strategien (Wissen) kreativ entwickelt, genutzt und verändert werden 
können. Das ist der körperphilosophische Zusammenhang, auf den James hingewiesen hat.

Darüber hinaus war ihm bereits klar, dass wir bestimmte introspektive Techniken der Körperwahrnehmung erlernen können, die es uns ermöglichen, die innere Verflechtung von muskulär-organischen, emotiv-motivationalen und geistigkognitiven Prozessen methodisch zu rekonstruieren. James hat diese Techniken aus experimentalpsychologischer Perspektive in den Blick gebracht (Shusterman, 2008, S. $161 \mathrm{ff}$.$) , dabei jedoch nur ihre theoretischen Implikationen anerkannt. Die$ praktischen und edukativen Potenziale, die in den Techniken der «somaesthetic introspection» (Shusterman, 2008, S. 158) für die gezielte Förderung kreativer Prozesse liegen, blieben bei ihm noch aussen vor. Erst Dewey hat in dieser Hinsicht den entscheidenden Schritt vollzogen.

Inspiriert von James' Pragmatismus hat Dewey dessen Perspektive auf die konkrete Praxis von Schule, Universität, Lebenswirklichkeit und Gesellschaft übertragen. Den von ihm entwickelten problembasierten Ansatz des learning by doing (Dewey, 1993) hat er im Rahmen seiner Chicagoer Laboratory School erfolgreich in der institutionellen Praxis erprobt. Zugleich ist Dewey hinsichtlich der Betonung des Körpers philosophisch konsequent über James' Ansatz hinaus gegangen. So hat er den bei James noch vorzufindenden Dualismus von Körper und Geist problematisiert. Der dualistischen Vorstellung zufolge handelt es sich bei Körper und Geist um zwei nicht nur graduell, sondern substanziell zu unterscheidende Entitäten. Alternativ dazu plädiert Dewey für eine graduelle und funktionale Differenzierung von körperlichen, seelischen und geistigen Prozessen:

Die Unterscheidung zwischen physisch, psycho-physisch und geistig ist also eine Unterscheidung von Ebenen anwachsender Komplexität und Intimität der Interaktion zwischen natürlichen Ereignissen. (Dewey, 1995, S. 252)

Durch diese antidualistische Kontinuitätsvorstellung vermeidet Dewey die bei James noch auftretenden reduktionistischen Tendenzen. Obwohl (bzw. gerade weil) Körper, Seele und Geist ein energetisches Kontinuum bilden, sind sie graduell und funktional zu differenzieren. Darauf weist auch Shusterman hin, wenn er schreibt:

Mind, in Dewey's view, is a still higher level of organization that emerges from psychophysical experience only when language comes into play, because language enables the organism's feelings and movements to be named, and thus objectified and given a determinate meaning that can be reidentified and deployed in communication. Mind remains in the realm of natural events, but Dewey's linguistic requirement for mind places it squarely in the realm of culture. (Shusterman, 2008, S. 186) 
Die Fundierung des Denkens als funktionales Element eines energetischen Aktivitätskontinuums, die sich bei Dewey auch in der Prägung von antidualistischen Bindestrichbegriffen wie «body-mind» (Dewey, 1984, S. 27) oder «mind-body» (Dewey, 1984 , S. 29) ausdrückt, hat Konsequenzen für ein ganzheitliches Verständnis des Lerngeschehens. Lernen erscheint aus Deweys Sicht als «product of the exercise of powers needed to meet the demands of the activity in operation» (Dewey, 1987, S. 238). Die kreative Lernerfahrung wird dabei nicht als "ein «Zusammenkommen» von Geist und Welt, Subjekt und Objekt, Methode und Stoff», sondern als «einheitlicher Prozess der Wechselwirkungen einer sehr grossen (...) Vielfalt von Kräften» (Dewey, 1993, S. 222) verstanden.

Noch deutlicher wird Dewey, wenn er an anderer Stelle den folgenden Vorschlag zur Begrifflichkeit macht: «[Within] the unity of behavior body stands for the means and agencies of conduct, and mind for its incorporated fruits and consequences» (Dewey, 1984, S. 31). Darin liegt die kulturpolitisch interessante, von Dewey aber nicht weiter verfolgte Idee, das Body-Mind-Vokabular durch ein Mittel-Zweck-Vokabular zu ersetzen. Vollzieht man diese Substitution, dann erscheint die Revalidierung des menschlichen Körpers in der modernen Bildungsforschung als Wiederentdeckung der praktischen Werkzeuge wirklichkeitsverändernden Handelns in der sozialen Interaktion mit anderen Menschen und der Natur (Gröschner \& Sandbothe, im Druck).

Für die Förderung von Kreativität im schulischen bzw. universitären Kontext hat die methodische Integration von Techniken der somatischen Introspektion aus Deweys Sicht grundlegende Bedeutung. Dewey selbst war für mehrere Jahrzehnte Schüler des australischen Schauspielers und Körperpädagogen Frederick M. Alexander (1869-1955). Sein Verständnis von kreativer Körperarbeit war sowohl durch diese intensiven praktischen Erfahrungen als auch durch die Lektüre von Alexanders Schriften geprägt, zu denen Dewey mehrere Vorworte und Einleitungen geschrieben hat (Dewey, 1982, 1983b, 1985).

Shusterman fasst die bildungsphilosophische Bedeutung von Alexanders edukativer Körperarbeit wie folgt zusammen:

The mass of kinaesthetic malfunctions and related somatic-psychic ailments (backaches, headaches, loss of vitality, nervousness, mental rigidity) that plague contemporary culture, Alexander explained as resulting from a systematic mismatch between our somatic tendencies developed through slow processes of evolution and the very different modern conditions of life and work in which we are forced to function. (Shusterman, 2008, S. 192f.) 
Und weiter bemerkt Shusterman:

Rejecting a regression to primitive life, he [i. e. Alexander] instead sought a method for people to rationally and consciously adjust their behavior to today's new and ever more quickly changing conditions (...) (Shusterman, 2008, S. 193)

Vor diesem Hintergrund erscheint Kreativität für Dewey in einem neuen, pragmatistischen Licht: «True spontaneity is henceforth not a birth-right but the last term, the consummated conquest, of an art - the art of conscious control» (Dewey, 1982, S. 352). Die methodische Kunst des Kreativen ergibt sich für Dewey aus dem, was Alexander «primäre Kontrolle des Gebrauchs des Selbst» (Alexander, 2001, S. 40) genannt hat. Sie besteht in einer bewusst ausbalancierten Haltung von Kopf und Nackenwirbelsäule im Verhältnis zum Rest des Körpers. Diese wiederum ist das schrittweise und allmähliche Produkt eines interaktiven Bewegungsbildungssprozesses. Dieser Prozess schliesst die gezielte Hemmung von Fehlgewohnheiten und die intelligente Reorganisation derjenigen habituellen Mittel ein, mit denen ein bestimmter motorischer Handlungszweck (wie z. B. Aufstehen, Hinlegen, Umdrehen, Gehen, Stehen, Sitzen) erreicht wird (Alexander, 2001).

Für Dewey handelt es sich bei Alexanders «Entdeckung einer zentralen Kontrolle» (Dewey, 2001, S. XVIII) um ein grundlegendes «Mittel zum Erlangen der vitalen Freiheit» (Dewey, 2001, S. XVIII), das den Einzelnen dazu befähigt, «durch eigenes koordiniertes Vermögen von seinem Potential Besitz zu ergreifen» (Dewey, 2001, S. XVIII). Es ist dieses Potenzial, in dem aus der Perspektive der pragmatistischen Körperphilosophie die kreative Fähigkeit beschlossen liegt, Neues zu Schaffen und damit die private Entwicklung des eigenen Lebens sowie den öffentlichen Fortschritt der menschlichen Kultur voran zu bringen. Aus diesem Grund folgert Dewey:

Alexanders Technik (...) bietet die Voraussetzung und ist daher zentral für alle spezifischen erzieherischen Prozesse. Sie steht im gleichen Verhältnis zu Erziehung, wie diese zu allen anderen menschlichen Handlungen steht. (Dewey, 2001, S. XVIII)

In seinem explizit von Alexander inspirierten Buch Human Nature and Conduct (1922) hat Dewey die folgende Definition von kreativer Aktivität vorgeschlagen: "Activity is creative as so far as it moves to its own enrichment as activity, that is, bringing along with itself a release of further activities» (Dewey, 1983a, S. 99). Wenn man diese Definition mit der besonderen Bedeutung zusammen denkt, die aus Deweys Sicht der primären Kontrolle des Gebrauchs des Selbst zukommt, er- 
gibt sich das Bild eines energetischen Tiefenzusammenhangs von Kreativität, Körperlichkeit und Bildung.

Eine sich selbst organisierende, sich aus sich selbst über sich hinaus treibende Aktivität setzt den freien Fluss und den intrinsisch motivierten Austausch zwischen somatischen, emotionalen und mentalen Lebensenergien voraus. Kreativität in Alexanders und Deweys Sinn gründet in eben dieser Erfahrung der freien Transformierbarkeit von körperlichen, seelischen und geistigen Prozessen ineinander. Aus pragmatistischer Perspektive ist die scheinbare Unerschöpflichkeit dieses Austauschs die entscheidende Quelle kreativer Schöpfungskraft und gehört aus diesem Grund ins Zentrum eines auf anspruchsvolle Weise demokratischen Bildungsverständnisses.

\section{Der Ansatz körperbasierten Lernens am Beispiel der Darstellung von zwei Seminarkonzepten}

Die bisherigen Ausführungen machen deutlich, dass der Körper eine grundlegende Rolle im Lehr- und Lernprozess einnehmen kann. Er bildet nicht nur auf physiologischer und neurobiologischer, sondern auch auf emotionaler, motivationaler und mentaler Ebene die energetische Basis für kreative Lehr- und Lernprozesse. Für den Unterricht ergibt sich daraus der Vorschlag, den Körper als mehrdimensionales Medium des Lehrens und Lernens gezielt zu integrieren und dadurch kreative Selbstregulationsprozesse in der Lehrpraxis auf neue Weise zu ermöglichen (Langer, 2008).

Erste empirische Befunde zur Stärkung von Selbstregulationskompetenzen durch körperbasiertes Lehren und Lernen liegen für die Lehrerausbildung vor. In ihrer Studie zur universitären Lehrerausbildung konnte Košinár (2007) zeigen, dass körperbasierte Selbstregulation durch expandierte Körperhaltung (also eine der Wachstumsbewegung folgende Streckung und Dehnung, die Alexanders primärer Kontrolle nicht unähnlich ist) ein wichtiges Instrument für die Stressprävention bei Lehramtsstudierenden darstellen kann und zugleich die Fähigkeit zur individuellen, situativen Selbststärkung positiv unterstützt (Košinár, 2007, S. 247ff.).

Im Folgenden stellen wir zwei von uns an Universitäten in Deutschland und Dänemark erprobte Seminarkonzepte vor, die den Ansatz körperbasierten Lernens in Aktion präsentieren. Im Rahmen des ersten Seminartypus kommt dem extrospektiven Einsatz von videobasierten Supervisionstechniken besondere Bedeutung zu (Stichwort: Körpersprache/nonverbale Ausdrucksfähigkeit). Innerhalb der zweiten Seminarform spielen demgegenüber die introspektiven Methoden kinästhetischer Bildung eine wichtige Rolle, die Frederick M. Alexander und Moshe Feldenkrais (1904-1985) entwickelt haben (Stichwort: Körperbewusstsein).

Beiden Veranstaltungskonzepten lag die folgende Arbeitsdefinition zugrunde: 
Körperbasiertes Lernen (oder auch body-based learning, kurz: BBL) ist ein Sammelbegriff für Lehrtechniken und Lernstrategien, die einen handlungsorientierten, ganzheitlichen und bewussten Umgang mit dem eigenen und fremden Körper unterstützen. BBL-Techniken intensivieren das balancierte Zusammenspiel von muskulär-organischen, emotiv-motiviationalen und mental-intellektuellen Ressourcen methodisch gezielt und helfen somit, die Kooperationsfähigkeit, Kreativität und moralische Sensibilität von Menschen zu stärken. Eine an BBL ausgerichtete Pädagogik betrachtet den Körper als mehrdimensionales Medium des Lehrens und Lernens in einer natürlichen, energetisch offenen und ökologisch sensiblen Umgebung, in der nachhaltige Erfahrungen gemacht und intelligente Formen der Interaktion eingeübt werden können.

\section{Seminarkonzeption I: Videobasierte Extrospektion}

Im Rahmen eines Seminars am erziehungswissenschaftlichen Institut der Universität Jena im WS 2007/08 mit dem Titel Kommunikation in Lehr- und Lernwelten wurde der Ansatz körperbasierten Lernens mit dem Fokus auf kommunikative Prozesse im Unterricht experimentell erprobt. ${ }^{1}$ Das Seminar gliederte sich in zwei Teile.

Im ersten Teil der wöchentlich stattfindenden Veranstaltungen wurden theoretische und methodische Aspekte der Kommunikation und Kommunikationspsychologie unter Bezugnahme auf für den Lehrerberuf wesentliche lernwirksame Dimensionen von Unterricht (z. B. Lehrer-Schüler-Beziehung, Unterrichtskommunikation, Klassenmanagement, Umgang mit Konflikten) erarbeitet und diskutiert. Darüber hinaus wurden methodische Kompetenzen, z. B. zu Aktivem Zuhören sowie zum Geben und Empfangen von Rückmeldungen kennengelernt und eingeübt; denn bedeutsam für das Gelingen des Ansatzes ist vor allem ein konstruktiver, wertschätzender Umgang unter den Teilnehmern. Dieser Seminarabschnitt wurde in der herkömmlichen Form von Referaten und Präsentationen der Lehramtsstudierenden durchgeführt, wobei sich für das Training der methodischen kommunikativen Fähigkeiten stets umfangreiche Diskussions- und Feedbackrunden in Kleingruppen und im Plenum an die Referate anschlossen.

Für den zweiten Teil des Seminars in Form einer mehrtätigen Blockveranstaltung ${ }^{2}$ wurden den Studierenden zuvor Arbeitsaufträge gegeben. Diese bestanden in der

1 Mittlerweile wurde der Ansatz auch in zahlreichen Workshops und Seminaren zum Erwerb pädagogischer Basiskompetenzen in der Lehrerausbildung an verschiedenen Universitäten und Hochschulen angewendet. Auch in schulinternen Lehrerfortbildungen stiess der Ansatz auf grosse Resonanz und konnte in längerfristigen Trainings- und Schulungskontexten ausgebaut werden (Gröschner, 2009).

2 Die Form von Blockveranstaltungen über einen bzw. zwei Tage mit je 8 Stunden hat nicht nur den gruppendynamischen Vorteil des besseren Kennenlernens und der grösseren Vertrautheit der Teilnehmerinnen und Teilnehmer untereinander, sondern ermöglicht eine erheblich intensivere inhaltliche Auseinandersetzung mit dem Gegenstand. Gerade die Arbeit mit Video(selbst)analysen hat ausserdem einen nicht zu unterschätzenden Zeitfaktor, für das, möchte man den grösstmöglichen Lernerfolg erreichen, das 90minütige Zeitkorsett herkömmlicher Seminarstrukturen nicht optimal ist. 
Regel darin, eine Stundeneröffnung (Umfang: 5 bis 10 Minuten) vorzubereiten. Diese konnten sie für eines der von ihnen studierten Unterrichtsfächer der Sekundarstufe frei wählen. Bereits diese Vorbereitungen zeigten ein hohes kreatives Potenzial der Studierenden, die erstmals in einer erziehungswissenschaftlichen Veranstaltung ihr unterrichtsbezogenens inhaltliches Fachwissen präsentieren konnten. Die Gruppe wurde auf die zur Verfügung stehenden Tage aufgeteilt, so dass kleinere Gruppen von zehn bis zwölf Lehramtsstudierende an dem Seminar teilnahmen.

An den jeweiligen Seminartagen wurden die einzelnen Präsentationen in Form von kurzen Rollenspielen (Lehrer/Lehrerin bzw. Klasse mit der von der Lehrperson zu bestimmender Klassenstufe und Unterrichtsstunde) vorgestellt und mit der Videokamera aufgezeichnet. ${ }^{3}$ Nach zwei bis drei Präsentationen wurden die kurzen Videosequenzen gemeinsam angeschaut und mit der Methode des "Lauten Denkens» (Van Someren et al., 1994; Weidle \& Wagner, 1994) bzw. des «call-out»/«stopping point»-Verfahrens (Frederiksen et al., 1998; Jacobs \& Morita, 2002) sowohl von der Seminarleitung als auch (zunehmend) von den Teilnehmerinnen näher analysiert. ${ }^{4}$ Dabei lag der Fokus vor allem auf verbalen/nonverbalen Aspekten des Verhaltens der angehenden Lehrperson. Als Ergebnis des Videospiegels können die folgenden Gesichtspunkte zusammenfassend festgehalten werden:

- Differenz von Selbst- und Fremdwahrnehmung: Ein Grossteil der Studierenden hat von sich ein anderes Selbstbild als es die Mitstudierenden zurückmelden;

- Reflexion von Körperhaltung, Mimik und Gestik: Die Studierenden geben häufig an, zuvor noch nie wirklich auf ihre Körperhaltung in der Unterrichtssituation (z. B. in Praktika) geachtet zu haben sowie teilweise Mimik und Gestik in dem gehaltenen Unterrichtseinstieg "unvorteilhaft» eingesetzt zu haben;

- Reflexion von Proxemik: Das Raumverhalten der Studierenden ist oftmals unvorteilhaft für den Austausch und den Blickkontakt mit den Schülern (vor allem unter Verwendung von Unterrichtsmedien wie Tafel, Beamer und Overheadprojektor);

- Reflexion von Paralinguistik: Insbesondere der verbale Ausdruck fällt den Studierenden auf. Dabei wird vor allem die Stimmführung (Prosodie) mehrmals kritisch angemerkt, z. B. wenn bei einem Arbeitsauftrag an die Schüler am Sat-

3 Erstaunlicherweise gaben Studierende, die im Rollenspiel Schülerinnen und Schüler einer Schulklasse darstellten, nach dem Seminar an, in bekannte Muster und Schülerrollen ihrer eigenen Schulzeit zurückgefallen zu sein. Dabei lag letztere zum Zeitpunkt des Seminars in den meisten Fällen schon mindestens 3 Jahre zurück. Es kann vermutet werden, dass eben diese bekannten Muster und Gewohnheiten einen wesentlichen Einfluss auf das eigene, spätere Handeln als Lehrkraft haben, werden die Erfahrungen nicht systematisch in der Ausbildung reflektiert (Messner, 2002).

4 Die zunehmende Aktivität der Diskussionsbeiträge und Analysen der Studierenden kann darauf zurück geführt werden, dass diese im Verlauf der Veranstaltung ihre eigene gegenstandsbezogene Wahrnehmung schulen konnten und ihnen daraufhin mehr und mehr Aspekte zur Rückmeldung aufgefallen sind. 
zende die Stimme der Lehrperson eher nach oben als nach unten geht (damit die Aufforderung eher wie eine Frage klingt) oder bei selbständigen Formen der Paralinguistik wie Lauten («Ähs» usw.).

Insgesamt geht aus den schriftlichen Rückmeldungen der Studierenden hervor, dass ihre Wahrnehmungs- und Reflexionskompetenz durch den Videospiegel erhöht werden konnte bzw. sie für ihr Selbstbild sensibilisiert worden sind. Das Auftreten der Studierenden, ihre Körpersprache, ihr verbaler Umgang mit den Schülern (die Lernbegleitung) konnte durch den Videospiegel und das Videofeedback genauer analysiert werden. Damit setzte ein Prozess der Bewusstheit ein, der - selbst in gespielten Situationen - persönlichkeitsorientiert das körperliche Verhalten zeigt. Die Blockveranstaltungen wurden nachträglich jeweils als grosse Bereicherung der Ausbildung bezeichnet. Dabei wurde vor allem die (Selbst)-Wahrnehmungsschulung als ein Element der Lehrerprofessionalisierung betont. Es wurde der Wunsch geäussert, diese Form des körperbasierten Lernens - insbesondere die Arbeit an Körperhaltung, Mimik, Gestik und Proxemik - als Element der Ausbildung zu intensivieren und zu verstetigen, da darin der Vorteil des Aufzeigens von individuellen Gewohnheiten sowie Möglichkeiten und Ratschläge zur Verbesserung (mit dem Ziel des kongruenten Zusammenspiels professionellen Wissens und individuellen Handelns) zum Tragen kommen können. ${ }^{5}$

Das kreative Potenzial, das mithilfe der videobasierten Extrospektion bei den Teilnehmerinnen und Teilnehmern freigelegt werden konnte, kann insbesondere in Form einer Ressourcen-Analyse beschrieben werden. Die Lernzeit sowie die Lernumgebung, der universitäre Ausbildungsort, wurden gezielt dazu genutzt, das individuelle unterrichtliche Handeln in Form eines Rollenspiels zu erproben und im angeleiteten Gruppenaustausch kriteriengeleitet zu reflektieren. Dabei wurden Aspekte des persönlichen Auftretens, der individuellen Kommunikation im Kontext der Vermittlung handlungsrelevanten Wissens (z. B. die Frage zum Umgang mit Konflikten im Klassenraum) und grundlegender Strategien (z. B. Wie gebe ich Rückmeldung?) ausgetauscht und dokumentiert.

Darüber hinaus gelang es durch den Fokus auf grundlegende, fächerübergreifende Merkmale des Lehrerhandelns, kreative Aspekte nicht im Sinne eines intellektuell eng geführten Unterrichtseinstiegs zu beschreiben. Vielmehr setzte der kommunikationsorientierte Videospiegel den Blick frei für ein integriertes Verständnis der

5 Ähnliche videobasierte Verfahren werden gegenwärtig in der amerikanischen Lehrerforschung systematisch integriert und effektiv genutzt. Jedoch wird in den Ansätzen des "Videoclub» (Sherin, 2007; Sherin \& van Es, 2009) bzw. «Problem-solving cycle» (Borko et al., 2008) weniger der Fokus auf körperbasiertes Lernen als vielmehr auf die Ausbildung einer reflexionsorientierten Veränderung in der Planung und Durchführung von Fachunterricht gelegt. Eine wünschenswerte Weiterentwicklung des Konzepts der videobasierten Extrospektion bestünde in der Hinsicht gerade in einer stärkeren Verknüpfung z. B. von fachdidaktischem Wissen mit dem pädagogischen Wissen und individuellen Auftreten. 
bedeutsamen Rolle des Körpers im Lehr- und Lernkontext. Dies wiederum führte zu einer höheren Bereitschaft, sich noch intensiver mit diesbezüglichen Professionalisierungsmassnahmen auseinanderzusetzen und sich dem kreativen Prozess der Kongruenz-Entwicklung stärker zu widmen. Im Verlauf des Seminars wurden somit nicht nur für die eigene Selbstregulation relevante Veränderungsbedürfnisse und Entwicklungsstrategien deutlich, sondern zugleich wurde dadurch auch die Wahrnehmung kreativitätsfördernder bzw. -hemmender Merkmale geschult.

\section{Seminarkonzeption II: Introspektion mittels Alexander-Technik und Feldenkrais-Methode}

Im Rahmen von Block-Seminaren und Workshops, die im Sommersemester 2004 an der Universität der Künste Berlin (UdK) sowie in den Jahren 2006 und 2007 an der Aalborg Universität Dänemark (AAU Campus Aalborg sowie AAU Campus Kopenhagen) und in der Werkstatt des dänischen Bildhauers Claus Ørntoft (Mygdal) statt gefunden haben, wurde ein komplementärer Ansatz körperbasierten Lehrens und Lernens erprobt (Sandbothe, 2010b). In Zusammenarbeit mit ausgebildeten Lehrerinnen der Alexander-Technik bzw. der Feldenkrais-Methode wurden Studierende in den Studiengängen "Gesellschafts- und Wirtschaftskommunikation» (an der Fakultät für Gestaltung der UdK Berlin) «Humanistische Informatik» (an der humanwissenschaftlichen Fakultät der AAU) und «Medialogie» (an der AAU-Fakultät für Technik, Wissenschaft und Medizin) bzw. im Rahmen des erwähnten MygdalWorkshops eingeladene Künstlerlnnen und Wissenschaftlerlnnen aus verschiedenen Fächern (www.mygdal.net) in die entsprechenden Ansätze und Techniken körperbasierten Lernens eingeführt.

Die Arbeit mit den Studierenden an den Universitäten erfolgte im Rahmen von Blockveranstaltungen, die im Regelfall als ein- oder mehrtägige Module von jeweils sechs Stunden (mit entsprechenden Pausen) angelegt waren. Die Studierenden befanden sich in einem Fall im Grundstudium (AAU Aalborg "Medialogie»), in den drei anderen in der Abschlussphase des Masterstudiums (UdK Berlin «Gesellschafts- und Wirtschaftskommunikation», AAU Aalborg «Humanistische Informatik», AAU Kopenhagen «Medialogie»).

Im Unterschied zu der an der Universität Jena durchgeführten Seminarkonzeption I setzten die Veranstaltungen in Aalborg, Berlin und Kopenhagen nicht bei der Aussenwahrnehmung, sondern bei der Innenwahrnehmung des eigenen Körperbildes an. Dementsprechend wurden Videoaufzeichnungen nur als Instrument der wissenschaftlichen Dokumentation verwendet, aber nicht als integraler medienpädagogischer Bestandteil der Veranstaltung eingesetzt. Stattdessen wurden die Studierenden von den erfahrenen und nach internationalen Standards zertifizierten Alexander- und Feldenkrais-Lehrerinnen in der bewussten Binnenwahrneh- 
mung (Introzeption) ihrer Muskulatur, ihrer Gelenke und ihres Skeletts im Wechselspiel mit der Schwerkraft in Raum und Zeit kinästhetisch geschult.

Im Unterschied zu den Jenaer Veranstaltungen hat sich gezeigt, dass bei dem beschriebenen Vorgehen die Aufmerksamkeit auf sogenannte Fehler kaum eine Rolle spielt. Das liegt daran, dass die externe Kontroll-Perspektive (Videospiegel) wegfällt und stattdessen die spielerische Erfahrung mit bewusster Muskelentspannung (Inhibition) und den sich daraus von selbst ergebenden innovativen Bewegungserfahrungen in den Vordergrund trat. Sowohl die Alexander-Technik als auch die Feldenkrais-Methode können jeweils entweder im One-to-One-Teaching eingesetzt werden oder als Gruppenarbeit. Von beiden Möglichkeiten wurde in den durchgeführten Lehrveranstaltungen in jeweils unterschiedlichen Kombinationen Gebrauch gemacht.

Eine ausführliche Beschreibung und Auswertung der im Rahmen dieser Veranstaltungen gesammelten BBL-Erfahrungen wird an anderer Stelle erfolgen (Sandbothe \& Gröschner, in Vorb.). Im aktuellen Kontext sei das Spannungsfeld, innerhalb dessen der bewusste Umgang mit dem eigenen Körper in aktuellen akademischen Ausbildungsinstitutionen konkret zu situieren ist, mit Hilfe einer anekdotischen Kurzgeschichte angedeutet, die zugleich zeigt, wie Kreativität durch den Einsatz von introspektiven Körperbewegungstechniken im Unterricht methodisch gestärkt werden kann.

Die im Folgenden nachgezeichnete Geschichte hat sich in einer Lehrveranstaltung ereignet, die 2007 auf dem AAU-Campus in Kopenhagen durchgeführt wurde. Es handelte sich um 15 fortgeschrittene (grösstenteils männliche) Studierende des stark technologisch geprägten Studiengangs Medialogie der Fakultät für Technik, Wissenschaft und Medizin der Aalborg Universität Kopenhagen.

Als die am Seminar beteiligte Alexanderlehrerin sich ans Pult stellte und die Studierenden ansprechen wollte, stellte sie fest, dass die meisten Studierenden von ihrer Anwesenheit kaum Notiz nahmen. Stattdessen verschwanden die Studenten mit ihren Körpern förmlich in den Laptops, die sie vor sich auf dem Tisch stehen hatten und aktiv mit Tastatureingaben versorgten. Um das Gespräch zu eröffnen, stellte die Alexanderlehrerin eine Frage, die sie direkt an denjenigen Studierenden richtete, dessen Körper am erfolgreichsten dabei war, in seinem mitgebrachten Laptop zu verschwinden. "Wie viele Stunden pro Tag verbringen Sie an Ihrem Computer?» Ein kurzes Aufsehen bei ununterbrochenem Tippen und beibehaltener Körperkrümmung. Keine Antwort.

Reformulierung der Frage: «Wie viel Zeit pro Tag verbringen Sie nicht am Computer?» Daraufhin sah der angesprochene Student kurz auf. Dieses mal mit gerichtetem Blick. Seine Aufmerksamkeit hatte sich von der Tastatur des Laptops abgewendet und verharrte für eine Sekunde zwischen Laptop und Handy, bevor sich der junge Mann geschäftsmässig der Tastatur seines Mobiltelefons zuwendete und diese ganz ähnlich traktierte wie zuvor den Laptop. In freier Übersetzung 
bedeutet diese performative Antwort auf die von der Alexanderlehrerin gestellte Frage soviel wie: «Ja, es gibt computerfreie Zeiten, z. B. dann wenn ich mich um mein Handy kümmere.»

Doch das war nur der erste Schritt zu einer Serie von Öffnungen, in die sich der erwähnte Studierende in den folgenden Stunden gemeinsam mit seinen Kommilitonlnnen unter Anleitung der Alexanderlehrerin begab. Dabei ging es darum, die Bewegungsmechanik des menschlichen Skeletts im Zusammenspiel mit der Muskulatur und unter Bedingungen der Schwerkraft anhand der eigenen Körpererfahrung zu spüren und zu optimieren. Bei der Feedback-Runde am Ende des Tages war der Laptop des erwähnten Marathontippers längst geschlossen und im Rücksack verstaut. Mit offenem Blick für seine Umgebung, in aufrechter und zugleich entspannter Haltung formulierte er sein Resümee wie folgt: «Am Anfang dachte ich, das ist bestimmt alles nur hypnotischer Hippie-Kram. Aber das stimmt nicht. Die Alexandertechnik ist total logisch. Warum haben wir das nicht schon im ersten Semester gelernt? Alextech kommt mir vor wie das Betriebssystem für meine mentale Software. Ich find's genial!»

Der Vergleich trifft den Grundmechanismus der Alexandertechnik sehr gut. Im Zentrum des von Alexander entwickelten und von Dewey körperphilosophisch weiter ausbuchstabierten Konzepts der primären Kontrolle steht unser Umgang mit unserer Wirbelsäule und dabei insbesondere die Art und Weise, wie wir unseren obersten Halswirbel nutzen. In Anspielung auf den Titanen Atlas aus der griechischen Mythologie wird er häufig auch als Atlasgelenk bezeichnet. Denn der in der klinischen Praxis als C1 titulierte Wirbel ist derjenige Wirbel, der dem Schädel am nächsten liegt, also den ganzen Kopf trägt und neben grundlegenden Balance-Funktionen auch Bewegungen wie das Nicken koordiniert. Zum Schädel hin ist das Atlasgelenk mit dem Hinterhauptsbein verbunden. Dabei handelt es sich um die anatomische Brückenstelle, an der Rückenmark und Gehirn ineinander übergehen.

Tatsächlich kommt in der Computerarchitektur dem Betriebssystem eine ähnliche Scharnierfunktion zu wie in der menschlichen Anatomie dem Atlasgelenk. Das operating system vermittelt zwischen hardware und software auf vergleichbare Weise wie im menschlichen Organismus das Atlasgelenk zwischen Gehirn und Bewegungsapparat. Wir neigen dazu, das Gehirn einseitig als Organ des Denkens zu verstehen. Zunächst aber dienen die basalen Gehirnfunktionen der Koordination von körperlichen Bewegungen, die über das Rückenmark an die entsprechenden Effektoren übermittelt werden. Wenn sich unser Atlasgelenk in einer entspannten und offenen Position befindet, dann erfolgt die Transformation von Gehirnimpulsen in körperliche Bewegungen auf geschmeidige und flexible Art und Weise. Ist unsere Nackenmuskulatur jedoch verspannt und dadurch das Atlasgelenk eingeklemmt und in seiner Bewegungsfreiheit behindert, dann ist auch der kreative Spielraum zwischen Gehirnimpuls und körperlicher Ausführung eingeschränkt. 
Interessanterweise ist es nun so, dass auch der umgekehrte Wirkungszusammenhang besteht. Wenn es uns gelingt mittels entsprechender Bewegungstechniken unsere Körperhaltung zu lockern, also unseren Umgang mit der Wirbelsäule so zu flexibilisieren, dass das Atlasgelenk eine entspanntere Position einnehmen kann, dann führt das in der mentalen Rückkopplung zu einer kreativen Öffnung unserer Geistestätigkeit, d. h. zu einer höheren Flexibilität im nicht länger nur konvergierenden, sondern in zunehmendem Masse dann auch divergenzfähigen Denken (Sandbothe, 2010b). Der erfahrene Alexanderpraktiker Dewey berichtet in diesem Zusammenhang «über die grosse Veränderung der moralischen und geistigen Einstellung, die eintritt, wenn sich die richtige Koordination etabliert hat» (Dewey, 2001, S. XVII).

Der dänische Medialogie-Student, der zunächst nicht bereit war von seinem Computer aufzuschauen, ist ein versierter Programmierer und hat viele Stunden seines erwachsenen Lebens mit gebeugter Kopfhaltung am Computer verbracht. Die Erfahrung, die er im Seminar mit der Alexandertechnik machen konnte, erlaubte es ihm, seine Nackenmuskulatur vorübergehend zu lockern und seinem Atlasgelenk mehr Spielraum zu lassen als gewohnt. Das führte zu einem Kreativitätsschub, den er dann auch für seine Programmierarbeit nutzen kann. Denn die Lockerung des Atlasgelenks, also die Optimierung der primären Kontrolle im Sinne von Alexander, führt zu eben derjenigen freien Transformierbarkeit von Energieflüssen zwischen körperlichen, seelischen und geistigen Prozessen, die Dewey als Grundgeschehen der kreativen Aktivität beschrieben hat.

\section{Ausblick}

Abschliessend kann festgehalten werden, dass die aufgezeigten Dimensionen körperbasierten Lernens in Zukunft in weiteren Seminaren noch detaillierter zu erproben sind. Auch sollte eine weiterführende Herangehensweise körperbasierten Lernens darin bestehen, die beschriebenen zwei Perspektiven - Extrospektion ( $\mathrm{Vi}$ deospiegel) und Introspektion (Alexander-Technik/Feldenkrais-Methode) - in gemeinsamen Workshops vernetzt zu erproben.

Darüber hinaus gilt es in empirisch angelegten Untersuchungen die Wirksamkeit des Ansatzes für Lehr-Lernprozesse zu dokumentieren und durch systematische Forschungen zu begleiten (z. B. im Kontext der Lehrerausbildung unter Rekurs auf fachdidaktische Professionalisierungsansätze). Unseres Erachtens bietet der Ansatz körperbasierten Lehrens und Lernens eine fruchtbare Perspektive für eine genauere Untersuchung, wenn es um die Frage geht, welche Rolle der Körper beim Lehren und Lernen spielt und wie durch eine gezielte Transformation bestehender Bewegungsabläufe und eine intelligente Schulung des propriozeptiven Systems (Todd, 1968, S. 26f.) Formen der Selbstwahrnehmung und des kreativen Selbstgebrauchs verbessert werden können. Auf dieser Grundlage könnte Bildung und 
Erziehung eine ganzheitliche Wirksamkeit entfalten, die das Aufwachsen im digitalen Zeitalter medienökologisch optimiert.

Vor mehr als 50 Jahren beschrieb Aldous Huxley den Zustand des westlichen Erziehungs- und Bildungssystems mit Blick auf dessen mangelndes Körperbewusstsein wie folgt:

Schooling without proper training of the psycho-physical instrument cannot, in the very nature of things, do more than a limited amount of good and may, in the process of doing that limited amount of good, do the child a great deal of harm by systematically engraining his habits of improper use. (Huxley, 1956, S. 21)

Und weiter schreibt Huxley:

The problem of incorporating a decent education in the non-verbal humanities into the current curriculum is a task for professional educators and administrators. What is needed at the present stage is research - intensive, extensive and long drawn research. Some Foundation with a few scores of millions to get rid of should finance a ten - or fifteen - year plan of observation and experiment. (Huxley, 1956, S. 38)

Möchte man Kinder und Jugendliche für den sensiblen Umgang mit Mensch, Technik und Natur befähigen, könnten nach unserer Auffassung in Zukunft zunehmend auch stärker präventive Massnahmen im Umgang mit dem eigenen Körper im Fokus des Lehrens und Lernens stehen. Bildungseinrichtungen wie Schulen und Hochschulen sind hierbei zentrale Orte der Vermittlung körperbasierter Medienkompetenz. Wie wir in den Ausführungen im Kontext der Initiierung und Stärkung von Kreativität gezeigt haben, interagieren körperliche Lehr- und Lernerfahrungen auf mehrdimensionale Art und Weise mit seelischen und geistigen Entwicklungsprozessen.

Um den Fortschritt von Bildung und Erziehung insgesamt voranzutreiben, bedarf es nicht nur der in vollem Gang befindlichen strukturellen Reformen im Bildungswesen und der gezielten Nutzung technologischer Innovationen, sondern auch einer umfangreichen empirischen Erforschung und parallel laufenden Integration von ganzheitlich kommunikations- und kooperationsorientierten sowie selbstreflektierten Lehr- und Lernmethoden. Zu diesem Zweck könnte der Ansatz körperbasierten Lernens zunächst selektiv und experimentell und dann möglicherweise auch flächendeckend in die schulische Unterrichtspraxis aufgenommen werden.

Der Mut, der dafür von Seiten der Lehrkräfte und Verantwortlichen in der Lehrerausbildung notwendig ist, hängt mit der Bereitschaft zusammen anzuerkennen, dass Lernprozesse existieren, die nicht in erster Linie kostengünstig als deklarati- 
ves Wissen standardisiert und vermarktet werden können. So schrieb Alfred North Whitehead einmal über die schöpferische Kraft der menschlichen Phantasie: «Imagination cannot be aquired once and for all, and then kept indefinitely in an ice box to be produced periodically in stated quantities. The learned and imaginative life is a way of living, and is not an article of commerce» (Whitehead, 1967, S. 97). In diesem Sinne gilt: Kreativität im Unterricht fördern bedeutet, auf die Lernenden einzugehen, ihre Voraussetzungen und Erfahrungen im Lernprozess ernst zu nehmen und als weiterführende intrinsische Lernanlässe zu verstehen. Zur Förderung von Kreativität gehört eine gelebte kreative pädagogische und didaktische Praxis der Lehrpersonen, zu der wir unter den Voraussetzungen lebensweltlicher Bezugspunkte im Unterricht eine Anknüpfung an Selbstregulationsprozesse durch körperbasiertes Lernen zählen können.

\section{Literatur}

Albert, Robert S. (1990): «Identity, experiences, and career choice among the exceptionally gifted and talented». Theories of creativity. Ed. by Mark A. Runco. Newbury Park: Sage, S. 13-34.

Alexander, Frederick M. (2001): Der Gebrauch des Selbst. Die bewusste Steuerung des Gebrauchs im Bezug auf Diagnose, Funktionieren und Reaktionskontrolle. Basel u. a.: Karger.

Borko, Hilda, Jacobs, Jennifer, Eiteljorg, Eric \& Pittman, Mary Ellen (2008): «Video as a tool for fostering productive discussions in mathematics professional development». Teaching and Teacher Education, 24, S. 417-436.

Bresler, Liora (Ed.) (2004): Knowing Bodies, Moving Minds. Towards Embodied Teaching and Learning. Dordrecht: Kluwer.

Cropley, Arthur (2001): «Kreativität und Kreativitätsförderung». Handwörterbuch Pädagogische Psychologie. Hrsg. von Detlef H. Rost. Weinheim: Beltz PVU, S. 366-373.

Csikszentmihalyi, Mihaly (1997): Kreativität. Stuttgart: Klett-Cotta.

Dewey, John (1982): «Introductory Word to F.M. Alexander's Man's Supreme Inheritance, in: The Middle Works: 1899-1924. Vol. 11: 1918-1919. Ed. by Jo Ann Boydston. Carbondale: Southern Illinois University Press, S. 350-353.

Dewey, John (1983a): Human Nature and Conduct, in: The Middle Works: 1899-1924. Vol. 14: 1922. Ed. by Jo Ann Boydston. Carbondale: Southern Illinois University Press.

Dewey, John (1983b): «Introduction to F. Matthias Alexander's Constructive Conscious Control of the Individual», in: Middle Works: 1899-1924. Vol. 15: 1923-1924. Ed. by Jo Ann Boydston. Carbondale: Southern Illinois University Press, S. 308-315. 
Dewey, John (1984): «Body and Mind», in: The Later Works: 1925-1953. Vol. 3: 1927-28. Ed. by Jo Ann Boydston. Carbondale: Southern Illinois University Press, S. 25-40.

Dewey, John (1985): «Introduction». In: F. M. Alexander: The Use of the Self. London: Orion Books, S. 7-12.

Dewey, John (1987): «What is learning?» in: The Later Works: 1925-1953. Vol. 11: 1935-1937. Ed. by Jo Ann Boydston. Carbondale: Southern Illinois University Press, S. 238-242.

Dewey, John (1988): Kunst als Erfahrung. Frankfurt a. Main: Suhrkamp.

Dewey, John (1993): Demokratie und Erziehung. Eine Einleitung in die philosophische Pädagogik. Weinheim und Basel: Beltz.

Dewey, John (1995): Erfahrung und Natur. Frankfurt a. Main: Suhrkamp.

Dewey, John (1997): How we think. New York: Dover.

Dewey, John (2001): «Einleitung», in: F. M. Alexander, Der Gebrauch des Selbst. Die bewusste Steuerung des Gebrauchs im Bezug auf Diagnose, Funktionieren und Reaktionskontrolle. Basel u. a.: Karger.

Egger, Rudolf (1996): Freiräume im Unterricht - Unterschätzt und überfordert? Kreativität als Erfahrungsquelle im schulischen Alltag. Innsbruck/Wien: Studien Verlag.

Feldenkrais, Moshe (1992): Das starke Selbst. Anleitung zur Spontaneität. Frankfurt a. M.: Suhrkamp.

Feldenkrais, Moshe (2005): Body and Mature Behavior. A Study of Anxiety, Sex, Gravitation and Learning, Berkeley: Frog Books.

Feldman, David Henry; Benjamin, Ann C. (2006): «Creativity and education: an American retrospective». Cambridge Journal of Education, 36(3), S. 319-336.

Frederiksen, John R.; Sipusic, Mike; Sherin, Miriam G.; Wolfe, Edward (1998): «Video portfolio assessment: Creating a framework for viewing the functions of teaching». Educational Assessment, 5(4), S. 225-297.

Friedmann, Elly D. (1989): Laban-Alexander-Feldenkrais. Pioniere bewusster Wahrnehmung durch Bewegungserfahrung, Paderborn: Junfermann.

Fudickar, Margrit (1985): Kreativitätstraining und Schule. Erfolgreiche Methoden zur Steigerung der Kreativität am Beispiel eines Unterrichtsversuchs. Essen: Verlag Die blaue Eule.

Giesecke, Michael (2002): Von den Mythen der Buchkultur zu den Visionen der Informationsgesellschaft. Trendforschungen zur kulturellen Medienökologie. Frankfurt/Main: Suhrkamp.

Giesecke, Michael (2005): «Auf der Suche nach posttypographischen Bildungsidealen». Zeitschrift für Pädagogik, 51(1), S. 14-29.

Giesecke, Michael (2007): Die Entdeckung der kommunikativen Welt. Studien zur kulturvergleichenden Mediengeschichte. Frankfurt/Main: Suhrkamp. 
Goleman, Daniel; Kaufman, Paul; Ray, Michael (1997): Kreativität entdecken. München/Wien: Hanser.

Glynn, Carol (2001): Learning On Their Feet. A Sourcebook for Kinesthetic Learning Across the Curriculum K-8. Shoreham: Discover Writing Press.

Gröschner, Alexander (2005): Pragmatische Medienkompetenz und Medienethik. Pädagogische Anknüpfungen an John Dewey im digitalen Zeitalter. Jena: IKS.

Gröschner, Alexander (2007): «Körpersprache im Unterricht. Perspektiven einer kommunikationsorientierten Bildungsforschung mithilfe von Unterrichtsvideos». Bildungsforschung, 4(2) URL: http://www.bildungsforschung.org/ bildungsforschung/Archiv/2007-02/koerpersprache [Zugriff: 7.4.09].

Gröschner, Alexander (2009): Videobasierte (Selbst-)Reflexion als Forschungsgegenstand: Eine explorative Studie in der Lehrerfortbildung von Berufsschullehrkräften. Vortrag auf dem Kongress "Unterrichtsforschung und Unterrichtsentwicklung» am 30. Juni 2009 an der Universität Zürich/Schweiz.

Gröschner, Alexander (im Druck): Innovation als Lernaufgabe.. Münster u. a.: Waxmann.

Gröschner, Alexander; Sandbothe, Mike (Hrsg.) (im Druck): Pragmatismus als Kulturpolitik. Beiträge zum Werk von Richard Rorty. Berlin: Suhrkamp.

Guilford, Joy Paul (1950): «Creativity». American Psychologist, 5, S. 445-454.

Guilford, Joy Paul (1970): «Creativity: retrospect and prospect». Journal of Creative Behavior, 5, S. 77-87.

Hannaford, Carla (2005): Smart Moves. Why Learning Is Not All In Your Head. Salt Lake City: Great River Books.

Hentig, Hartmut von (1998): Kreativität. Hohe Erwartungen an einen schwachen Begriff. München \& Wien: Hanser.

Hoffmann, Bernward (2003): Medienpädagogik. Eine Einführung in Theorie und Praxis. Paderborn: Schöningh.

Huxley, Aldous (1956): Adonis and the Alphabet. And other Essays. London: Chatto \& Windus.

Jacobs, Jennifer K.; Morita, Eiji (2002): «Japanese and American Teachers' Evaluations of Videotaped Mathematics Lessons». Journal for Research in Mathematics Education, 33(3), S. 154-175.

James, William (1970): The Principles of Psychology. New York: Dover.

James, William (1976): Essays in Radical Empiricism. Cambridge (MA): Harvard University Press.

James, William (1983): Talks to Teachers on Psychology and to Students on some of Life's Ideals. Cambridge: Harvard University Press.

Jeffrey, Bob (2006): «Creative teaching and learning: towards a common discourse and practice». Cambridge Journal of Education, 36(3), S. 399-414. 
Košinár, Julia (2007): Selbststärkung im Lehrerberuf: Individuelle und kontextuelle Bedingungen für die Anwendung körperbasierter Selbstregulation. Baltmannsweiler: Schneider Verlag Hohengehren.

Langer, Antje (2008): Disziplinieren und entspannen. Körper in der Schule - eine diskursanalytische Ethnographie. Bielefeld: transcript.

Loi, Daria; Dillon, Patrick (2006): «Adaptive educational environments as creative spaces». Cambridge Journal of Education, 36(3), S. 363-381.

Mathews, Ann (1984): Implications for Education in the Work of F.M. Alexander. An Explanatory Project in a Public School Classroom. New York: Institute for Research, Development, and Education in the Alexander Technique.

Messner, Rudolf (2002): "Schule als Lernort im Spannungsfeld von Theorie und Praxis der LehrerInnenbildung». Lehrerinnen- und Lehrerbildung braucht Qualität. Und wie! Hrsg. von Hans Brunner, Erich Mayr, Michael Schratz \& Ilsedore Wieser. Innsbruck: Studien-Verlag, S. 59-79.

Neff, Günter (1975): «Kreativität und Gruppe». Kreativität in Schule und Gesellschaft. Voraussetzungen, didaktische Modelle und Perspektiven. Hrsg. von Günter Neff. Ravensburg: Otto Maier Verlag, S. 72-102.

Pikler, Emmi (2001): Lasst mir Zeit. Die selbständige Bewegungsentwicklung des Kindes bis zum freien Gehen. München: Pflaum.

Preiser, Siegfried; Buchholz, Nicole (2004): Kreativität. Ein Trainingsprogramm für Alltag und Beruf. Heidelberg: Asanger.

Reinmann, Gabi (2005): «Innovation ohne Forschung? Ein Plädoyer für den DesignBased Research-Ansatz in der Lehr-Lernforschung». Unterrichtswissenschaft, 33(1), S. 52-69.

Reinmann, Gabi; Mandl, Heinz (2006): «Unterrichten und Lernumgebungen gestalten». Pädagogische Psychologie. Ein Lehrbuch. Hrsg. von Andreas Krapp und Bernd Weidenmann. Weinheim: Beltz, S. 613-658.

Renkl, Andreas (2006): «Träges Wissen: Wenn Erlerntes nicht genutzt wird». Psychologische Rundschau, 47, S. 78-92.

Renkl, Andreas (2008): «Lernen und Lehren im Kontext der Schule». Lehrbuch Pädagogische Psychologie. Hrsg. von Andreas Renkl. Bern: Huber \& Hogrefe, S. 109-153.

Sandbothe, Mike (2003): «Der Vorrang der Medien vor der Philosophie». Medienphilosophie. Beiträge zur Klärung eines Begriffs. Hrsg. von Stefan Münker, Alexander Roesler und Mike Sandbothe. Frankfurt a. M.: Fischer, S. 185-197.

Sandbothe, Mike (2005): «Einleitung: Wozu systematische Medienphilosophie?» Systematische Medienphilosophie. Hrsg. von Mike Sandbothe und Ludwig Nagl. Berlin: Akademie-Verlag, S. XIII-XXVII.

Sandbothe, Mike; Nagl, Ludwig (Hrsg.) (2005): Systematische Medienphilosophie. Berlin: Akademie-Verlag. 
Sandbothe, Mike (2010a): «Und wie geht es Ihrem Nacken?» Kulturaustausch. Zeitschrift für internationale Perspektiven, Themenheft: e-volution, 3/2010, S. 2223 (Online-Ausgabe: http://www.ifa.de/pub/kulturaustausch/archiv/ausgaben2010/e-volution/und-wie-fuehlt-sich-ihr-nacken-an/ [Zugriff: 22.07.2010].)

Sandbothe, Mike (2010b): «Berufliche Erfahrungen als Hochschullehrer mit der Feldenkrais-Methode und der Alexandertechnik in Deutschland, Dänemark und Finnland». Online-Publikation: http://www.sandbothe.net/847.html [Zugriff: 01.06.2010].

Sandbothe, Mike; Gröschner, Alexander (Hrsg.) (in Vorb.): Body-based Learning. Ein pragmatistischer Ansatz für eine medienökologische Bildungskultur. Wiesbaden: VS Verlag für Sozialwissenschaften.

Scheerens, Jaap; Bosker, Roel J. (1997). The foundations of educational effectiveness. Oxford: Pergamon Press.

Seidel, Tina; Prenzel, Manfred; Schwindt, Katharina; Rimmele, Rolf; Kobarg, Mareike; Dalehefte, Inger Marie (2009). «The Link between Teaching and Learning - Investigating Effects of Physics Teaching on Student Learning in the Context of the IPN Video Study». The Power of Video Studies in Investigating Teaching and Learning in the Classroom. Hrsg. von Tomas Janik und Tina Seidel. Münster: Waxmann, S. 161-180.

Serve, Helmut J. (1995): Förderung der Kreativitätsentfaltung als implizite Bildungsaufgabe der Schule. München: PimS-Verlag.

Sherin, Miriam G. (2007): "The Development of Teachers' Professional Vision in Video Clubs». Video Research in the Learning Sciences. Eds. by Ricki Goldman, Roy Pea, Brigid Barron \& Sharon J. Derry. Mahwah, N. J.: Erlbaum, S. 383-395.

Sherin, Miriam G.; van Es, Elisabeth (2009): «Effects of Video Club Participation on Teachers' Professional Vision». Journal of Teacher Education, 60(1), S. 20-37.

Shelav-Silberbusch, Chava (1999): Bewegung und Lernen. Die Feldenkrais-Methode als Lernmodell. Dortmund: Verlag modernes Lernen.

Shuell, Thomas J. (1996): «Teaching and learning in a classroom context». In David C. Berliner \& Robert C. Calfee (Eds.), Handbook of educational psychology. New York: Simon \& Shuster Macmillan, S. 726-764.

Shusterman, Richard (2008): Body Consciousness. A Philosophy of Mindfulness and Somaesthetics. Cambridge: Cambridge University Press.

Sternberg, Robert J.; Lubart, Todd (1999): «The concept of creativity: prospects and paradigms». Handbook of creativity. Ed. by Robert J. Sternberg. Cambridge: Cambridge University Press, S. 3-15.

Todd, Mabel. E. (1968): The Thinking Body. A Study of the Balancing Forces of Dynamic Man. Hightstown: Princeton Book Company.

Van Someren, Maarten W.; Barnard, Yvonne F.; Sandberg, Jacobijn A. C. (1994): The think aloud method: A practical guide to modelling cognitive processes. London u. a.: Academic Press. 
Weidle, Renate; Wagner, Angelika C. (1994): «Die Methode des Lauten Denkens». Verbale Daten. Eine Einführung in die Grundlagen und Methoden der Erhebung und Auswertung. Hrsg. von Günter L. Huber und Heinz Mandl. Weinheim/Basel: Psychologie-Verlags-Union, S. 81-103.

Weiss, Sabine (2008): «Kreativitätsförderung». Unterricht sehen, analysieren, gestalten. Hrsg. von Ewald Kiel. Bad Heilbrunn: Klinkhardt, S. 119-141.

Whitehead, Alfred N. (1929/1967): The aims of Education and other essays. New York: Free Press. 